\title{
Über den Einfluß der Startlage auf die Stoffwanderung bei der Papierelektrophorese $\left.{ }^{1}\right)$
}

\author{
Von G. SCHNEIDER und G. SCHNEIDER \\ C. u. O. Vogt-Institut für Hirnforschung, Neustadt/Schwarqwald (Direktor: Prof. Dr. A. Hopf)
}

(Eingegangen am 10. März 1967)

Die Lage des Startpunktes bei der Papierelektrophorese ist nicht nur bedeutend für die Wanderungsgeschwindigkeit, sondern auch für die Wanderungstichtung von Substanzen. Aus Gründen der Reproduzierbarkeit sollte man daher bei der Beschreibung papierelektrophoretischer Versuche nicht versäumen, stets die Lage der Auftragsstelle anzugeben.

Versuche mit Wechselspannungen ergaben, daß die Umkehr der Wanderungsrichtung, die bei vielen Substanzen in Abhängigkeit von der Lage des Startpunktes auftritt, nicht allein auf die Strömungswanderung zurückgefühtt werden kann.

The starting position in paper electrophoresis not only affects the rate, but also the direction of migration of substances. Therefore, for the sake of reproducibility, the position of application should always be quoted in a description of paper electrophoresis experiments.

Experiments with reversed currents showed that the change in the direction of migration, which for many substances depends upon the starting position, is not entirely dependent upon the direction of the current.

Bei papierelektrophoretischen Analysen ergaben sich immer wieder Unregelmäßigkeiten der Wanderungsgeschwindigkeit und -richtung der untersuchten Stoffe, obwohl die Versuchsbedingungen weitgehend konstant gehalten wurden (1). Als eine mögliche Variable erschien bei der Papierelektrophorese die Lage der Auftragsstelle, die man ja im allgemeinen so wählt, daß eine möglichst große Wanderungsstrecke zur Verfügung steht (2).

Um zu klären, ob zwischen Stoffwanderung und Startlage ein Zusammenhang besteht, wurden Substanzen an verschiedenen Stellen des Trägerpapieres aufgetragen und ihre elektrophoretische Wanderung ermittelt.

Dazu wurde jeweils eine Substanz von 7 verschiedenen Positionen aus auf 7 Einzelstreifen $(30 \mathrm{~cm}$ lang) gestartet. Die Position I lag dabei $9 \mathrm{~cm}$ von dem einen Streifenende (Kathode), die Startposition VII $9 \mathrm{~cm}$ vom anderen Streifenende (Anode) entfernt. Die restlichen Positionen II bis VI waren in gleichen Abständen zwischen den Positionen I und VII verteilt. Um möglichst konstante Versuchsbedingungen $\mathrm{zu}$ erhalten, wurden alle Streifen eines Versuches gleichzeitig in eine Elektrophoresekammer (nach GrasSMANN u. HANNIG, modifiziert (3)) eingelegt.

Die Versuche ergaben, daß für alle geprüften Substanzen die Wanderungsgeschwindigkeit stets von der Lage des Startpunktes abhängt (Abb. 1). Das wurde für alle untersuchten Spannungen zwischen 110 und $1500 \mathrm{~V}$ gefunden. Aus Abbildung 1 wird ferner deutlich, daß bei einigen Substanzen auch eine Beziehung zwischen Startposition und Wanderungsrichtung besteht. Die Abhängigkeit der elektrophoretischen Wanderungsrichtung von der Startlage ermöglicht eine Einteilung der untersuchten Stoffe in 3 Gruppen:

1. Stoffe, die von jeder Startposition aus nur anodisch wandern (z. B. Humanalbumin).

2. Substanzen, die abhängig von der Stattlage anodisch oder kathodișch wandern, wie: $\alpha$-Alanin, Arginin, Asparagin, Cystein, L-Glutamin, Glutaminsäure, Glykokoll, L-Leucin, Methionin-HCl, Serin, Taurin, Tryptophan und Glucose.

1) Mit Unterstützung der Deutschen Forschungsgemeinschaft.

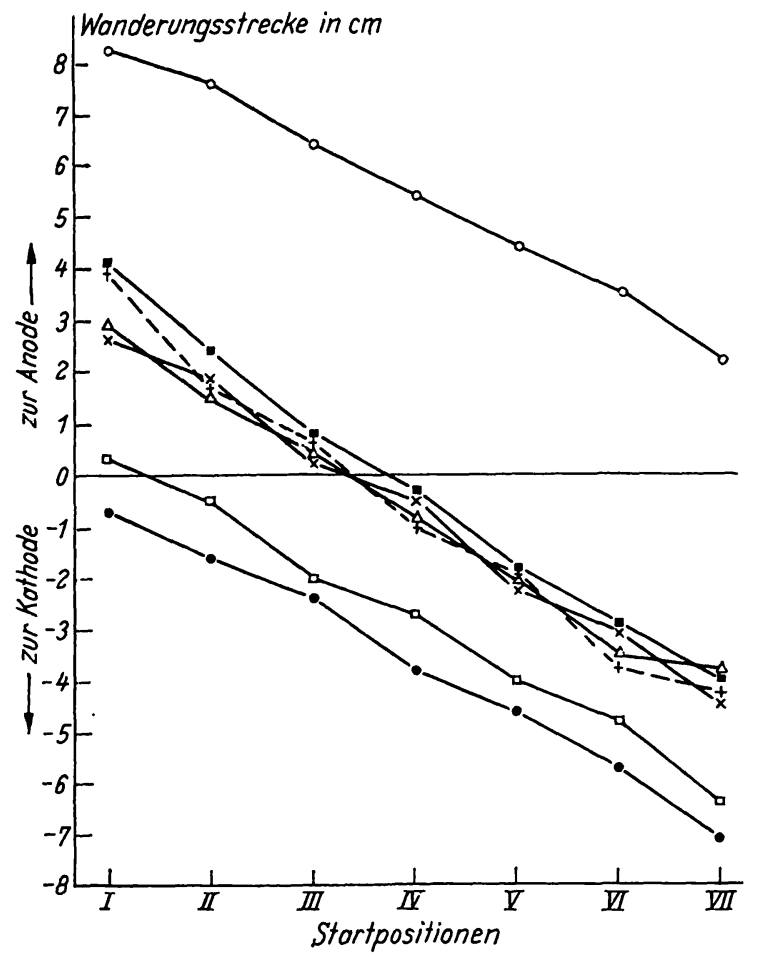

Abb. 1

Die Abhängigkeit der papierelektrophoretischen Wanderung verschiedener Substanzen von der Lage des Starts.

○ـ Humanalbumin (Veronalpuffer, $\mathrm{pH}=8,6$. Verdünnung 1:3,

$+\cdots+$ Glucose (Borat-Natronlauge-Puffer, $\mathrm{pH}=11,5$, Ver$+\cdots+$ dünnung $1:-100,1500 \mathrm{~V}, 15 \mathrm{Min}$.)

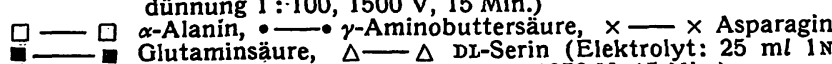
Essigsäure in $500 \mathrm{ml}$ Aqua dest., $1050 \mathrm{~V}, 15 \mathrm{Min}$.)

3. Stoffe, die von jeder Startposition aus nur kathodisch wandern (z. B.: $y$-Aminobuttersäure, Histidin- $\mathrm{HCl}$ und Lysin-HCl).

Auffallend ist, daß die aus Startlage und Wanderungsstrecke resultierenden Kurven (Abb. 1) aller geprüften Substanzen etwa parallel zueinander verlaufen, unabhängig von der angelegten Spannung (110-1500 V), der Versuchsdauer und der Art des verwendeten Elektrolyten. Dabei ist es gleichgültig, ob die sich bei höheren Spannungen bildende JounEsche Wärme durch orga- 
nische Lösungsmittel abgeleitet wird (4), oder ob man es durch Verdünnen des Puffers gar nicht erst zu übermäßiger Wärmebildung kommen läßt (5).

Aus entsprechenden Versuchen geht hervor, daß der elektrophoretische Trenneffekt von der Lage des Starts unabhängig ist. Darauf deuten bereits die Kurven in Abbildung 1 hin, die alle praktisch parallel zueinander verlaufen.

Wie bereits dargestellt, können zahlreiche Stoffe bei der Elektrophorese ihre Wanderungsrichtung umkehren (6). Es ist unbekannt, wie dieser Umkehreffekt zustande kommt. Zunächst galt zu prüfen, ob die im Papier infolge Wärmeentwicklung entstehende Pufferströmung vielleicht für diesen Effekt verantwortlich zu machen ist.

Um eine Vorstellung über die Größe des Pufferstromes in Abhängigkeit von der Spannung zu bekommen, wurde Asparagin in verschiedenen Positionen aufgetragen und eine Wechselspannung an die Streifen angelegt. Dabei kommt es zu keiner elektrophoretischen - Wanderung der Substanz. Es tritt aber eine Pufferströmung auf, die eine Wanderung des aufgetragenen Stoffes bewirkt (Strömungswanderung). Pufferstrom und Strömungswanderung dürften bei Wechselspannung von gleicher Größenordnung sein, wie sie auch bei Anlegen eines entsprechenden Gleichstromfeldes entstehen. In einem Versuch wurde an die Streifen eine Wechselspannung von $110 \mathrm{~V}$ für $160 \mathrm{Min}$. angelegt, in einem weiteren Experiment eine Wechselspannung von $880 \mathrm{~V}$ für 20 Min. Aus Abbildung 2 geht hervor, daß die

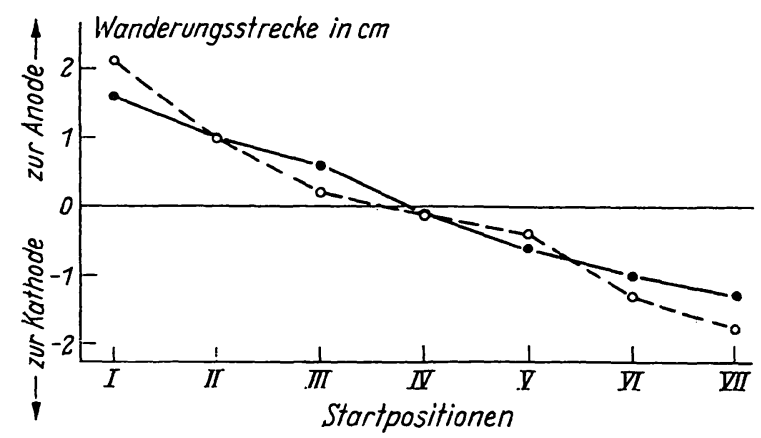

Abb. 2

Strömungswanderung von Asparagin in Abhängigkeit von der Lage des Starts. Elektrolyt: $25 \mathrm{~m} l$ 1N Essigsäure in $500 \mathrm{~m} l$ Aqua dest.

- - $110 \mathrm{~V}$ Wechselspannung, $160 \mathrm{Min}$. Versuchsdauer $\circ-\circ 880 \mathrm{~V}$ Wechselspannung, 20 Min. Versuchsdauer

Stoffwanderung durch die Pufferströmung im Papier bei einer angelegten Wechselspannung von $880 \mathrm{~V}$ und $20 \mathrm{Min}$. Versuchsdauer genau der von $110 \mathrm{~V}$ Wechselspannung bei 160 Min. Versuchsdauer entspricht. Wie zu erwarten, ist also die durch die Pufferströmung bewirkte Wanderung von Substanzen bei der Papierelektrophorese direkt proportional der angelegten Spannung. Sie steigt auch bei $n$-facher Spannung in einer Versuchszeit von $1 / \mathrm{n}$ nicht über den $\mathrm{n}$-fachen Wert an. Das gilt für alle Startpositionen.
Aus Abbildung 2 geht ferner hervor, daß die Strömungswanderung von Substanzen in den elektrodennahen Startlagen am größten ist und sich in Richtung auf die Streifenmitte hin vermindert. In der Streifenmitte ist sie praktisch gleich Null. Die Strömungswanderung erfolgt stets zur Mitte des Streifens hin. Ganz entsprechend der Wanderung hat man sich die Stärke des Pufferstromes vorzustellen. Diese letztgenannten Befunde stehen in guter Übereinstimmung mit den Untersuchungen von MICHL (7), bei denen eine Wechselspannung von $300 \mathrm{~V}$ verwendet würde.

Trotz dieser Ergebnisse kann noch nicht ausgeschlossen werden, daß bei Anwendung höherer Spannungen und entsprechend verstärkter Pufferströmung die Wanderungsrichtung hier nicht doch mehr beeinflußt wird als im Bereich geringer Spannungen.

Entsprechende Versuche wurden ebenfalls mit Asparagin durchgeführt, das von den verschiedenen Positionen aus gestartet wurde. Im ersten Versuch wurde eine Gleichspannung von $900 \mathrm{~V}$ für $20 \mathrm{Min}$. an die Streifen angelegt, im zweiten Experiment bei gleicher Versuchsdauer eine Wechselspannung von $900 \mathrm{~V}$ benutzt. Die Wanderungswerte für das Asparagin in Abhängigkeit von Startlage und Art der Spannung sind aus $A b$ bildung $3 \mathrm{zu}$ ersehen. Hier ist auch das Verhältnis der Strömungswanderung zur Gesamtwanderung (Wanderung durch Pufferstrom + reine elektrophoretische Motilität) deutlich zu erkennen. Das genannte Verhältnis ist von Startposition zu Startposition verschieden. Es beträgt z. B. bei Position III 1:11, bei Position V dagegen nur 1:1. Diese Ergebnisse gelten im Prinzip auch für Versuche mit geringeren Spannungen. Der Anteil der durch die Pufferströmung bedingten Stoffwanderung an der Gesamtmotilität der Substanzen ist unabhängig von det zur Elektrophorese benutzten Spannung.

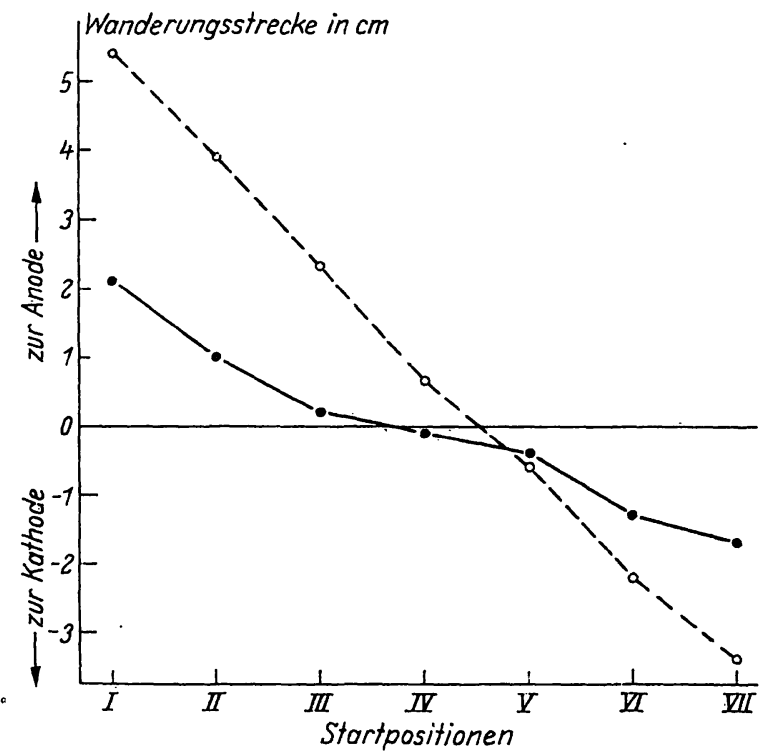

Abb. 3

Strömungswanderung $-\longrightarrow(900 \mathrm{~V}$ Wechselspannung, $20 \mathrm{Min}$. Versuchsdauer) und Gesamtwanderung $\circ \longrightarrow 0$ (900 V Gleichspannung, $20 \mathrm{Min}$. Versuchsdauer) von Asparagin in Abhängigkeit von der Lage des Starts. Elektrolyt: $25 \mathrm{ml}$ 1N Essigsäure in $500 \mathrm{ml}$ Aqua dest. 
Daraus ergibt sich, daß auch unter extremen Bedingungen die Umkehr der Wanderungsrichtung nicht allein von der Strömungswanderung abhängen kann. Der Pufferstrom verläuft bei denjenigen Substanzen, die ihre Wanderungsrichtung in Abhängigkeit von der Startlage umkehren, stets in der gleichen Richtung wie die wan- dernde Substanz, bleibt aber in der Mehrzahl der Startpositionen stark hinter dem Wert für die Gesamtwanderung zurück. Alle Substanzen, die ihre Wanderungsrichtung nicht.umkehren, wandern von einigen Startpositionen aus sogar dem Pufferstrom entgegen.

\title{
Literatur
}

1. GRAF, E. und P. H. List, Naturwissenschaften 40, 273 (1953). -

2. Clotten, R. und A. Clotren, Hochspannungselektrophorese, Georg Thieme Verlag, Stuttgart (1962). - 3. Grassmann, W. und K. HanNig, Hoppe-Scyler's Z. physiol. Chem. 290, 1 (1952). -
4. Mrchl, H., Mh. Chem. 82, 489 (1951). - 5. Schneider, G. und G. SPARMANN, Naturwissenschaften 42, 156 (1955). - 6. v. Holt, L., K. D. Vorgt und K. GAEDE, Biochem. Z. 323, 345 (1952). 7. MrCHL, H., Mh. Chem. 85, 1251 (1954).

\section{Mikromethode zur enzymatischen Bestimmung von Acetessigsäure und D-(-)- $\beta$-Hydroxybuttersäure in Blut und Geweben}

\author{
Von G. Stein und K. H. BÄsSLER \\ Aus dem Pbysiologisch-Chemischen Institut der Universität Mainz (Direktor: Prof. Dr. R. K. Zabn)
}

(Eingegangen am 9. Mai 1967)

Es wird eine Mikromethode zur enzymatischen Bestimmung von Acetessigsäure und $\beta$-Hydroxybuttersäure in Blut und Geweben beschrieben. Bei Patienten mit Ketoacidose und bei hungernden Ratten können beide Ketonkörper in $0,1 \mathrm{~m} l$ Blut bestimmt werden. Für die Bestimmung normaler Spiegel beim Menschen benötigt man je $0,2 \mathrm{~m} l$ Blut für Acetessigsäure und $\beta$-Hydroxybuttersäure. Daten über die Genauigkeit der Methode, Wiederfindungsversuche und Werte in Rattenblut und menschlichem Blut werden mitgeteilt.

A micromethod is described for the enzymic determination of acetoacetic acid and $\beta$-hydroxybutyric acid in blood and tissues. In patients with keto-acidosis and in starved rats, both ketone bodies can be determined in $0.1 \mathrm{~m} l$ of blood. $0.2 \mathrm{~m} l$ of blood is required for the measurement of normal levels of acetoacetic acid and $\beta$-hydroxybutyric acid in humans. Data are given for the accuracy of the method, the efficiency of recovery and values from rat blood and human blood.

Für Untersuchungen des Ketonkörper-Stoffwechsels sind wir auf eine Methode angewiesen, die es erlaubt, in $0,1 \mathrm{~m} l$ Rattenblut Acetessigsäure und $\beta$-Hydroxybuttersäure zu bestimmen. Ausreichende Spezifität gewährleistet nur die enzymatische Bestimmung. BERGMEYER und BERNT (1) haben eine enzymatische Methode beschrieben, die aus der Methode von Williamson, Meldandy und KREBS (2) entwickelt ist. Für Untersuchungen an kleinen Versuchstieren erfordert sie aber zuviel Blut. Wir haben diese Methode für eine Mikrobestimmung modifiziert, so daß bei hungernden Ratten beide Ketonkötper in $0,1 \mathrm{~m} l$ Blut zuverlässig bestimmt werden können. Um Normalwerte beim gesunden Menschen messen zu können, benötigt man je $0,2 \mathrm{~m} l$ Blut für Acetessigșäure und $\beta$-Hydroxybuttersäure. Für die Untersuchung pathologischer Fälle und für die Verlaufskontrolle von Ketoacidosen genügen auch beim Menschen $0,1 \mathrm{~m} l$ Blut für die Bestimmung beider.Ketonkörper, da hierbei die untere Nachweisgrenze bei Werten erreicht wird, die nicht mehr als pathologisch bezeichnet werden können. $\mathrm{Da}$ man bei Mikrolitermethoden trotz geringeren Enzymverbrauchs höhere Enzymkonzentrationen im Ansatz hat als bei Makromethoden, verringert sich der Zeitbedarf für eine Bestimmung wesent- lich. Allerdings fallen deshalb auch Fremdaktivitäten im Enzympräparat stärker ins Gewicht und müssen berücksichtigt werden.

\section{Methodik}

Prinzip der Methode

3-Hydroxybutyrat-Dehydrogenase ${ }^{1}$ ) katalysiert die Dehydrierung von $\beta$-Hydroxybuttersäure mit NAD ${ }^{2}$ ) bzw. die Reduktion von Acetessigsäure mit NADH. Dieser Vorgang wird nach dem Prinzip der Endwertbestimmung bei $366 \mathrm{~nm}$ registriert. $\mathrm{Da}$ käufliche Präparate von 3-Hydroxybutyrat-Dehydrogenase noch Lactat- und Malat-Dehydrogenase enthalten, müssen vor der Bestimmung von Acetessigsäure Proben, die Pyruvat und Oxalacetat enthalten, mit Lactatdehydrogenase und Malatdehydrogenase inkubiert werden, um diese Substanzen zu beseitigen. Im Gegensatz zu den Angaben von BERGMEYER (1) fanden wir eine solche Vorinkubation auch bei der Bestimmung von $\beta$-Hydroxybuttersäure notwendig, besonders, wenn die Lactatkonzentration in den Proben groß war. Der Grund

1) Der Trivialname 3-Hydroxybutyrat-Dehydrogenase wird hier gebraucht für das Enzym D-3-Hydroxybutyrat: NAD-Oxydoreduktase EC I. I. I. 30; Lactat-Dehydrogenase für L-Lactat: NAD-Oxydoreduktase EC I.I.I. 27; Malatdehydrogenase für L-Malat: NAD-Oxydoreduktase EC I. I. I. 37.

2) Abkürzungen: $\mathrm{NAD} \mathrm{bzw} . \mathrm{NADH}=$ Oxydiertes bzw. reduziertes Nicotinamid-Adenin-Dinucleotid; $\mathrm{LDH}=$ Lactatdehydrogenasc; $\mathrm{MDH}=$ Malatdehydrogenase; 3- $\mathrm{HBDH}=3$-Hydroxybutyratdehydrogenase. 